\title{
Electron- and proton-impact excitation of heliumlike uranium in relativistic collisions
}

\author{
A. Gumberidze, ${ }^{1}$ D. B. Thorn, ${ }^{2}$ A. Surzhykov, ${ }^{3,4}$ C. J. Fontes, ${ }^{5}$ B. Najjari, ${ }^{6}$ A. Voitkiv, ${ }^{7}$ S. Fritzsche,${ }^{8}{ }^{9}$ D. Banaś, ${ }^{10}$ \\ H. F. Beyer, ${ }^{11}$ W. Chen, ${ }^{12}$ R. E. Grisenti, ${ }^{11}, 13$ S. Hagmann, ${ }^{11,13}$ R. Hess, ${ }^{11}$ P.-M. Hillenbrand, ${ }^{11}$ P. Indelicato, ${ }^{14}$ \\ C. Kozhuharov, ${ }^{11}$ M. Lestinsky, ${ }^{11}$ R. Märtin, ${ }^{8,11}$ N. Petridis, ${ }^{11}$ R. V. Popov,${ }^{15}$ R. Schuch, ${ }^{16}$ U. Spillmann, ${ }^{11}$ S. Tashenov, ${ }^{17}$ \\ S. Trotsenko, ${ }^{8,11}$ A. Warczak, ${ }^{18}$ G. Weber, ${ }^{8,11}$ W. Wen, ${ }^{6}$ D. F. A. Winters, ${ }^{11}$ N. Winters, ${ }^{11}$ Z. Yin, ${ }^{19}$ and Th. Stöhlker ${ }^{8,11,20}$ \\ ${ }^{1}$ ExtreMe Matter Institute EMMI and Research Division, GSI Helmholtzzentrum für Schwerionenforschung, D-64291 Darmstadt, Germany \\ ${ }^{2}$ Lawrence Livermore National Laboratory, Livermore, California 94550-9234, USA \\ ${ }^{3}$ Physikalisch-Technische Bundesanstalt, D-38116 Braunschweig, Germany \\ ${ }^{4}$ Technische Universität Braunschweig, D-38106 Braunschweig, Germany \\ ${ }^{5}$ Computational Physics Division, Los Alamos National Laboratory, Los Alamos, New Mexico 87545, USA \\ ${ }^{6}$ Institute of Modern Physics, Chinese Academy of Sciences, Lanzhou 730000, China \\ ${ }^{7}$ Institute for Theoretical Physics I, Heinrich-Heine-University of Düsseldorf, D-40225 Düsseldorf, Germany \\ ${ }^{8}$ Helmholtz-Institut Jena, D-07743 Jena, Germany \\ ${ }^{9}$ Theoretisch-Physikalisches Institut, Friedrich-Schiller-Universitt Jena, D-07743 Jena, Germany \\ ${ }^{10}$ Institute of Physics, Jan Kochanowski University, PL-25-406 Kielce, Poland \\ ${ }^{11}$ GSI Helmholtzzentrum für Schwerionenforschung, D-64291 Darmstadt, Germany \\ ${ }^{12}$ China Spallation Neutron Source (CSNS), Chinese Academy of Sciences, Institute of High Energy Physics, Dongguan 523803, China \\ ${ }^{13}$ Institut für Kernphysik, Universität Frankfurt, D-60486 Frankfurt am Main, Germany \\ ${ }^{14}$ Laboratoire Kastler Brossel, Sorbonne Université, CNRS, ENS-PSL Research University, Collège de France, \\ Case 74, 4, place Jussieu, F-75005 Paris, France \\ ${ }^{15}$ Department of Physics, St. Petersburg State University, St. Petersburg 198504, Russia \\ ${ }^{16}$ Physics Department, Stockholm University, S-106 91 Stockholm, Sweden \\ ${ }^{17}$ Physikalisches Institut, Ruprecht-Karls-Universität Heidelberg, D-69120 Heidelberg, Germany \\ ${ }^{18}$ Institute of Physics, Jagiellonian University, PL-30-348 Krakow, Poland \\ ${ }^{19}$ Laboratory for Physical Chemistry, ETH Zürich, CH-8093 Zürich, Switzerland \\ ${ }^{20}$ IOQ, Friedrich-Schiller-Universität Jena, D-07743 Jena, Germany
}

(Received 19 December 2018; published 13 March 2019)

\begin{abstract}
We have studied the $K$-shell excitation of He-like uranium $\left(\mathrm{U}^{90+}\right)$ in relativistic collisions with hydrogen and argon atoms. Performing measurements with different targets, as well as with different collision energies, enabled us to explore the proton- (nucleus-) impact excitation as well as the electron-impact excitation process for the heaviest He-like ion. The large fine-structure splitting in uranium allowed us to partially resolve excitation into different $L$-shell levels. State-of-the-art relativistic calculations which include excitation mechanisms due to the interaction with both protons (nucleus) and electrons are in good agreement with the experimental findings. Moreover, our experimental data clearly demonstrate the importance of including the generalized Breit interaction in the treatment of the electron-impact excitation process.
\end{abstract}

DOI: 10.1103/PhysRevA.99.032706

\section{INTRODUCTION}

One of the fundamental processes playing an important role in collisions involving highly charged ions (HCI) is the excitation of an electron bound to the ion. Collisions between a highly charged ion and a light atom resulting in the excitation of the ion are often characterized by momentum transfers that are much larger than the typical momenta of the atomic electrons. It has been shown that in this case

Published by the American Physical Society under the terms of the Creative Commons Attribution 4.0 International license. Further distribution of this work must maintain attribution to the author(s) and the published article's title, journal citation, and DOI. the target electrons and nucleus can be considered as acting independently in the collision process [1,2]. This has been also nicely demonstrated experimentally by ionizing light ions in collisions with neutral atoms [3-6]. Consequently, projectile excitation in fast asymmetric collisions can be described as a sum of two independent processes: proton- (or nucleus-) impact excitation (PIE) and electron-impact excitation (EIE). The cross sections for the two processes scale as $Z_{T}^{2}$ and $Z_{T}$, respectively $\left(Z_{T}\right.$ being the target atomic number) $[1,2]$. Therefore, the relative contribution of the two processes is different for different targets, e.g., the relative contribution of EIE would be largest for the hydrogen target, whereas for heavy targets the excitation will be dominated by the contribution due to the target nucleus.

Heavy-ion storage rings, such as the Experimental Storage Ring (ESR) at GSI in Darmstadt, equipped with internal gas 
targets have provided very favorable conditions to extend the former studies of the excitation process for low- and mid- $Z$ ions ([7-15]) into the high- $Z$ regime. Namely, the excitation of projectile electrons due to the interaction with the target nucleus (PIE) has been addressed in detail for very heavy hydrogen- and heliumlike ions (see, e.g., Refs. [16-18]). In Ref. [18], specifically the nuclear-field-induced excitation of $\mathrm{H}-$ and He-like uranium ions was studied. A markedly different behavior observed for the two systems could be explained by rigorous relativistic predictions emphasizing the importance of the magnetic interaction and many-body effects in the strong-field domain. In these studies, targets such as $\mathrm{N}_{2}$ or heavier have been used, making the nuclear-impact excitation the dominant process.

Owing to the development of a new multiphase target for the ESR $[19,20]$ providing densities of up $10^{15}$ particles $/ \mathrm{cm}^{2}$, we were able to explore for the first time the EIE of H-like uranium [21]. Here, it is important to emphasize that EIE is a fundamental atomic process which plays a very prominent role in various kinds of laboratory and astrophysical plasmas. Most of the experimental studies of the EIE process for HCI have been performed with the electron beam ion trap (EBIT) devices limited, for the case of excitation, to the mid- $Z$ regime [22-24]. In order to gain access to the EIE process for H-like uranium, in Ref. [21] we performed measurements with different targets, namely, $\mathrm{H}_{2}$ and $\mathrm{N}_{2}$, as well as with different collision energies, 212.9 and $393.9 \mathrm{MeV} / u$. The study has shown that the effect of EIE is clearly visible when one compares the projectile excitation for $\mathrm{N}_{2}$ and $\mathrm{H}_{2}$ targets. Namely, EIE is prominent for the case of $\mathrm{H}_{2}$ target, due to the above-mentioned scaling of the nuclear and electron contributions $\left(Z_{T}^{2}\right.$ and $\left.Z_{T}\right)$. Moreover, the experimental results have clearly demonstrated the importance of the generalized Breit interaction (GBI) in the EIE process.

In this paper, we present an extension of our previous study [21] for the $K$-shell excitation (PIE and EIE) of heliumlike uranium. The obtained results enable a stringent and detailed test of the state-of-the-art relativistic calculations which include excitation mechanisms due to both protons (nucleus) and electrons.

The paper is structured as follows: In the next section, the experimental arrangement as well as the experimental method is described, and in Sec. III, we then present the data analysis and compare our experimental results with the predictions of a fully relativistic theory. In addition, a relation of the current results for He-like uranium with those obtained for H-like uranium in our earlier study [21] is discussed. In Sec. IV, finally, a short summary is given, together with some ideas on possible future extensions of the current study.

\section{EXPERIMENT}

The experiment followed a very similar scheme as in Ref. [21]. Around $10^{8} \mathrm{He}$-like uranium ions produced by successive acceleration and stripping were stored and cooled in the ESR. For the measurement, the internal supersonic jet target was used, crossing the beam in a perpendicular direction. The target areal densities were between $10^{13}$ and $10^{14}$ particles $/ \mathrm{cm}^{2}$ and the interaction zone was defined by an overlap of the cooled ion beam (diameter $2 \mathrm{~mm}$ ) with the

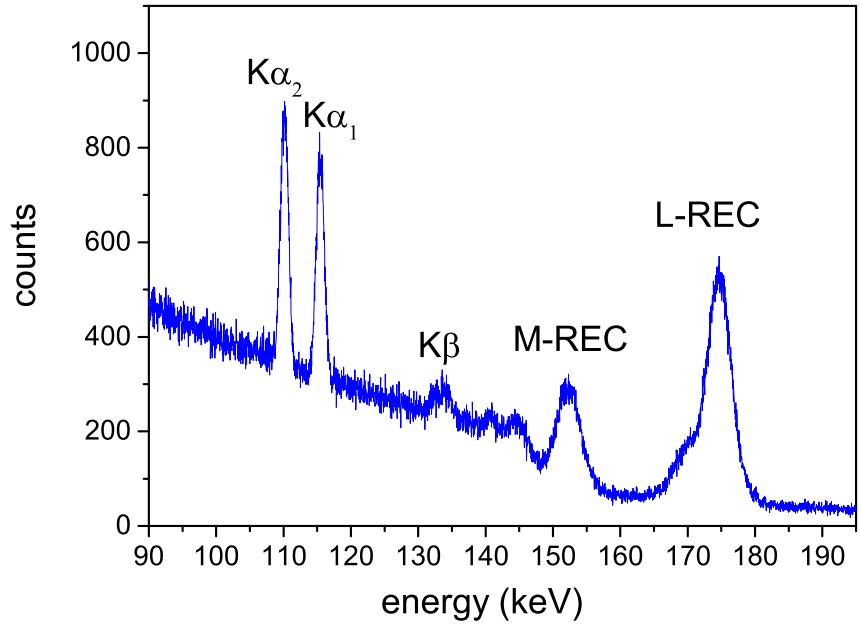

FIG. 1. X-ray spectrum recorded for $218-\mathrm{MeV} / u \mathrm{U}^{90+} \rightarrow \mathrm{H}_{2}$ collisions with a $\mathrm{Ge}(\mathrm{i})$ detector at the observation angle of $60^{\circ}$ with respect to the ion beam. The REC transitions into $L, M$, and higher shells are clearly visible together with the characteristic transitions into the $K$ shell.

jet target (diameter $6 \mathrm{~mm}$ ). The experiment was performed for $\mathrm{H}_{2}$ and $\mathrm{Ar}$ targets and two different beam energies of 218 and $300 \mathrm{MeV} / u$. These energies were chosen to be near and well above the EIE threshold. The corresponding kinetic energies for equivelocity electrons in the rest frame of the ions are 119.6 and $164.8 \mathrm{keV}$, respectively, and the $K-L$ excitation energies are in the range of $96-101 \mathrm{keV}$ for He-like uranium. The projectile excitation process was explored by looking at characteristic $\mathrm{x}$ rays emitted during the decay of the excited $L$-shell levels. For this purpose, we used the atomic physics experimental chamber at the internal target of the ESR. Here, projectile $\mathrm{x}$ rays produced in collisions of the stored ion beams with the jet target were detected by an array of Ge(i) detectors, covering observation angles in the range between $35^{\circ}$ and $150^{\circ}$ with respect to the beam axis. The solid angles covered by the individual detectors were on the order of $10^{-3}$. The photon detectors were energy and efficiency calibrated before the experiment using a set of appropriate radioactive sources. The projectile ions that captured an electron were detected after the next dipole magnet of the ESR with a multiwire proportional counter (MWPC). More details concerning the experimental setup at the ESR jet target can be found in Refs. [17,18,25] and references therein.

In Fig. 1 we present, as an example, the x-ray spectrum for $\mathrm{U}^{90+} \rightarrow \mathrm{H}_{2}$ collisions at $218 \mathrm{MeV} / u$ recorded at the observation angle of $60^{\circ}$ with respect to the ion beam direction. In the x-ray spectrum, broad lines associated with the radiative electron capture (REC) of the target electron into the projectile $L$ and $M$ shells are clearly visible. In addition, the characteristic $K \alpha$ and $K \beta$ lines on top of the background (stemming from bremsstrahlung emission by target electrons deflected in the field of the projectile nucleus) are prominent. Since in the current experiment we are dealing with He-like projectiles, i.e., ions with a closed $K$ shell, the $K \alpha$ and $K \beta$ lines are a direct signature of the $K$-shell excitation. In contrast to $\mathrm{H}$-like projectiles [21], here the $K \alpha$ and $K \beta$ lines cannot be produced by an electron capture into excited levels and a 
subsequent decay into the $K$ shell. In principle, a simultaneous excitation and ionization of the projectile $K$ shell could be possible as it has been observed in previous studies [26]; however, first, the corresponding cross section is very small, especially for light targets, and, second, this process would produce Lyman lines of H-like uranium which would be readily resolved from the $K \alpha$ lines of He-like uranium by our detectors [21]. No Lyman transitions could be identified in our spectra for all the cases. Therefore, in a further analysis we focus on the $K \alpha$ lines to gain insight into the excitation process of He-like uranium. Here, $K \alpha_{1}$ and $K \alpha_{2}$ lines comprise $\left[1 s_{1 / 2}, 2 p_{3 / 2}\right]{ }^{1} P_{1},\left[1 s_{1 / 2}, 2 p_{3 / 2}\right]{ }^{3} P_{2} \rightarrow\left[1 s^{2}\right]{ }^{1} S_{0}$ and $\left[1 s_{1 / 2}\right.$, $\left.2 p_{1 / 2}\right]{ }^{3} P_{1},\left[1 s_{1 / 2}, 2 s_{1 / 2}\right]{ }^{3} S_{1} \rightarrow\left[1 s^{2}\right]{ }^{1} S_{0}$ transitions in Helike uranium, respectively. These pairs of transitions cannot be resolved by our detectors.

\section{EXPERIMENTAL RESULTS AND COMPARISON WITH THEORY}

Similarly to our previous study for H-like uranium [21], here we concentrate on the intensity ratios of the $K \alpha_{1}$ and $K \alpha_{2}$ lines. The number of counts recorded in the $K \alpha_{1}$ and $K \alpha_{2}$ spectral lines were determined by fitting the corresponding peaks in the spectra with Gaussian functions on top of a linear background due to bremsstrahlung. As a next step, the intensity ratios were corrected for the energy-dependent detector efficiency. For this correction, an error of $3 \%$ is included.

The $K \alpha_{1} / K \alpha_{2}$ intensity ratios as a function of the observation angle can be described by the following formula [27,28],

$$
W\left(\theta_{\mathrm{lab}}\right)=R \frac{1+B_{1}\left(1-\frac{3}{2} \frac{\sin ^{2} \theta_{\mathrm{lab}}}{\gamma^{2}\left(1-\beta \cos \theta_{\mathrm{lab}}\right)^{2}}\right)}{1+B_{2}\left(1-\frac{3}{2} \frac{\sin ^{2} \theta_{\mathrm{lab}}}{\gamma^{2}\left(1-\beta \cos \theta_{\mathrm{lab}}\right)^{2}}\right)} .
$$

Here, $\theta_{\text {lab }}$ is the photon observation angle with respect to the beam direction in the laboratory frame. $\beta$ and $\gamma$ are the relativistic factors corresponding to the particular beam energy. The angular emission patterns of the $K \alpha_{1}$ and $K \alpha_{2}$ spectral lines are determined by the effective anisotropy parameters $B_{1}$ and $B_{2}$ which are in turn related to the alignment of the corresponding magnetic sublevels whose exact form depends on the transition under consideration. As already mentioned, for the excitation of He-like uranium, all four levels, $\left[1 s_{1 / 2}, 2 p_{3 / 2}\right]{ }^{1} P_{1},\left[1 s_{1 / 2}, 2 p_{3 / 2}\right]{ }^{3} P_{2},\left[1 s_{1 / 2}, 2 p_{1 / 2}\right]{ }^{3} P_{1}$, $\left[1 s_{1 / 2}, 2 s_{1 / 2}\right]{ }^{3} S_{1}$, can be in principle populated and thus contribute to the $K \alpha_{1}$ and $K \alpha_{2}$ transitions. Therefore, it is not possible to obtain the alignment parameters for these individual states by fitting Eq. (1) to the angular distribution of the $K \alpha_{1} / K \alpha_{2}$ intensity ratios. This is in contrast to the H-like case where it was possible to extract directly the alignment parameter for the $2 p_{3 / 2}$ state from the angular distribution of the $\mathrm{Ly} \alpha_{1}$ line [18,29]. The parameter $R$ gives the $K \alpha_{1} / K \alpha_{2}$ intensity ratio at the "magic angle," i.e., the angle for which the angle-dependent part of Eq. (1) is zero.

In order to obtain the experimental values for the $K \alpha_{1} / K \alpha_{2}$ intensity ratios, we fitted their angular distributions with Eq. (1) allowing $R, B_{1}$, and $B_{2}$ to be free parameters. In Fig. 2, the results of this fit procedure are shown for the collision energy of $218 \mathrm{MeV} / u$. From the figure, one can see that the

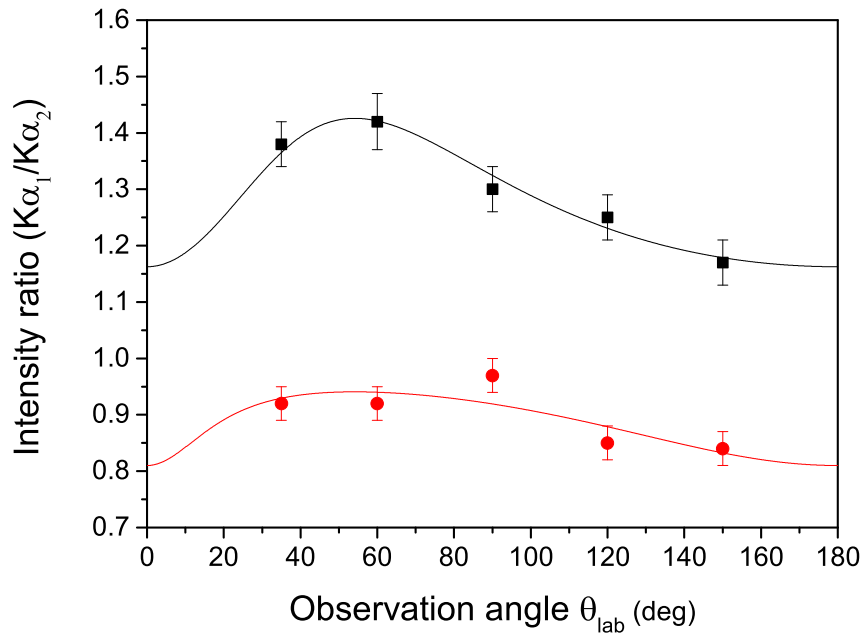

FIG. 2. The $K \alpha_{1} / K \alpha_{2}$ intensity ratios as a function of the observation angle for $218-\mathrm{MeV} / u \mathrm{U}^{90+}$ collisions with $\mathrm{Ar}$ (black squares) and $\mathrm{H}_{2}$ targets (red circles). The solid lines refer to corresponding fits of Eq. (1) to the data (see also text).

$K \alpha_{1} / K \alpha_{2}$ intensity ratios are clearly smaller (by $\sim 30 \%$ ) for the case of collisions with $\mathrm{H}_{2}$ target as compared to Ar. This finding is very similar to the one from our earlier study on the excitation of H-like uranium [21].

In order to compare our experimental results for the $K \alpha_{1} / K \alpha_{2}$ intensity ratios with theory, we need the total cross sections for excitation (PIE as well as EIE) to the levels contributing to the respective spectral lines. In addition, the decay scheme of He-like uranium has to be taken into account. As can be seen in Fig. 3, $\left[1 s_{1 / 2}, 2 p_{3 / 2}\right]{ }^{3} P_{2}$ and $\left[1 s_{1 / 2}, 2 p_{1 / 2}\right]{ }^{3} P_{0}$ levels have two decay branches each. As a result, the deexcitation from the $\left[1 s_{1 / 2}, 2 p_{3 / 2}\right]{ }^{3} P_{2}$ state contributes to both $K \alpha_{1}$ and $K \alpha_{2}$ lines and the $\left[1 s_{1 / 2}, 2 p_{1 / 2}\right]{ }^{3} P_{0}$ state contributes to the $K \alpha_{2}$ line via the $E 1$ decay branch to the $\left[1 s_{1 / 2}, 2 s_{1 / 2}\right]{ }^{3} S_{1}$ state. The direct decay from the $\left[1 s_{1 / 2}, 2 p_{1 / 2}\right]{ }^{3} P_{0}$ state to the ground state proceeds via two-photon emission (E1M1)

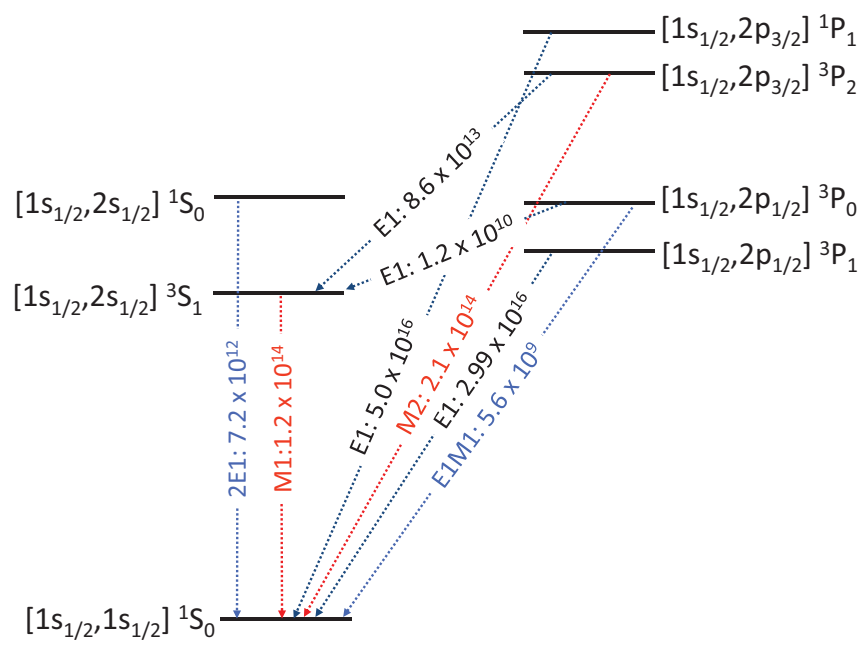

FIG. 3. Level scheme and main decay modes of the first excited levels in He-like uranium. The corresponding multipolarities and transition rates [30] (given in units of $\mathrm{s}^{-1}$ ) are indicated as well. 

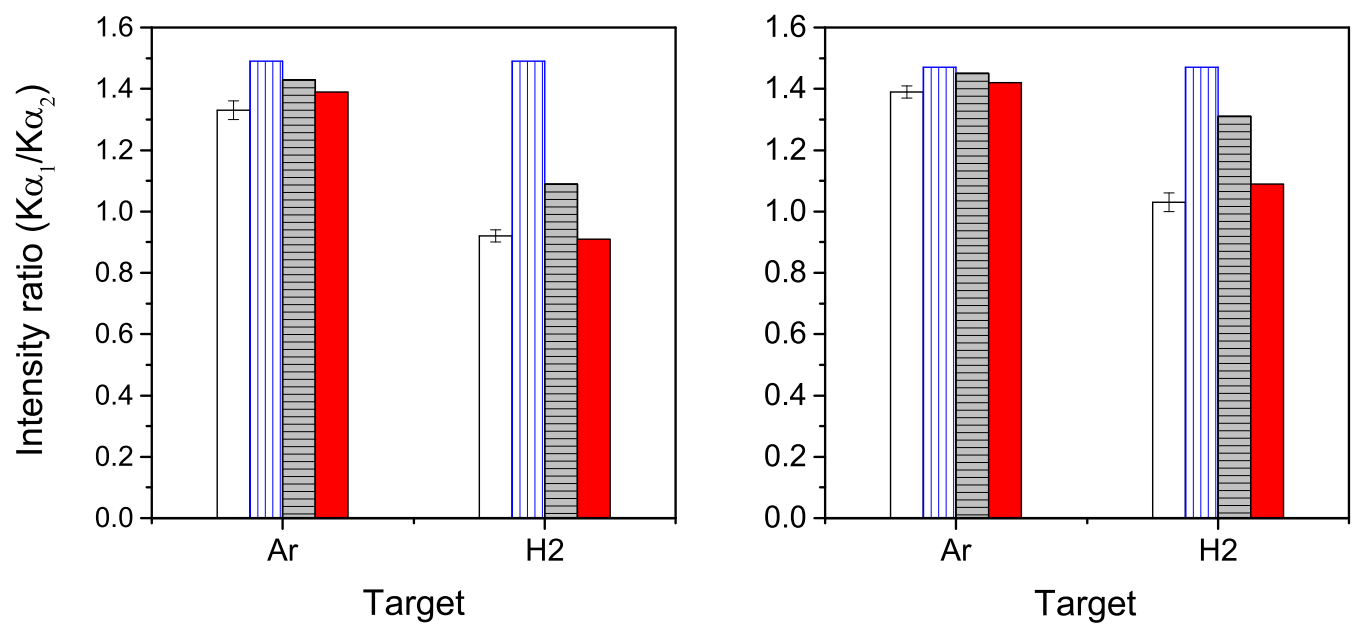

FIG. 4. Experimental results (white columns) in comparison with theoretical predictions for $K \alpha_{1} / K \alpha_{2}$ ratios $\left(A_{0}\right)$ for the K-shell excitation of $\mathrm{U}^{90+}$ in collisions with $\mathrm{Ar}$ and $\mathrm{H}_{2}$ targets at 218 (left) and $300 \mathrm{MeV} / u$ (right). Blue columns with vertical stripes show PIE results. Solid red columns depict combined (PIE+EIE) calculations. In addition, the combined calculations without inclusion of the GBI are presented by gray columns with horizontal stripes.

and thus it does not contribute to the $K \alpha$ lines with similar considerations for the two-photon decay (2E1) from the $\left[1 s_{1 / 2}, 2 s_{1 / 2}\right]{ }^{1} S_{0}$ state. Taking this into account, the theoretical $K \alpha_{1} / K \alpha_{2}$ intensity ratios can be calculated as follows,

$$
R=\frac{\sigma\left({ }^{1} P_{1}\right)+0.71 \sigma\left({ }^{3} P_{2}\right)}{\sigma\left({ }^{3} P_{1}\right)+0.29 \sigma\left({ }^{3} P_{2}\right)+\sigma\left({ }^{3} S_{1}\right)+0.68 \sigma\left({ }^{3} P_{0}\right)} .
$$

Here, the $\sigma(\cdots)$ refers to the population cross section of the corresponding level and the numbers are the branching ratios for the corresponding transitions based on the transitions rates from [30]. This formula includes the excitation only to $n=2$ states and the subsequent decay.

In Fig. 4, we present a comparison of our experimental results for $K \alpha_{1} / K \alpha_{2}$ intensity ratios with theoretical predictions. The experimental uncertainties include contributions due to statistics and the detector efficiencies. The theoretical predictions include both excitation processes, PIE and EIE, assuming (quasi)free electrons. This approximation is justified in this case due to the fast asymmetric collisions involving large momentum transfers [2]. The theoretical results also include excitation to higher levels $(n>2)$ and subsequent cascade contributions to the observed $K \alpha$ radiation. However, the cascade contributions to the $K \alpha_{1} / K \alpha_{2}$ intensity ratios turn out to be quite small, on the order of $1 \%$. Furthermore, the effect of the Compton profile of the target electron on the EIE cross sections has been estimated to be negligible in the case of the $\mathrm{H}_{2}$ target which is of primary relevance for investigating the EIE process. It should be noted that for the Ar target, being not of primary interest for the EIE process, even though the effect of the Compton profile due to $K$-shell electrons is quite large, the overall effect due to contributions from the $L$ - and $M$-shell electrons is still at the level of only a few percent.

The PIE calculations were performed within the framework outlined in Ref. [31]. In this approach, the electron-nucleus interaction is treated in the first-order perturbation theory and (initial and final) states of helium-like ions are described by the multiconfiguration Dirac-Fock (MCDF) method. The validity of this approach for our collision regime has been demonstrated in earlier studies $[17,18]$. The EIE cross-section calculations are based on a relativistic distorted-wave approach $[2,32]$ including the effects of the GBI. Here, we would like to note that the GBI can be derived as a lowest-order quantum electrodynamics (QED) correction to the electronelectron interaction and its importance has already been verified for a few atomic processes [34-37].

From the comparison of our experimental data with the theory it can be seen that the calculations including only the PIE process cannot reproduce the experimental results. The deviation is particularly pronounced for the $\mathrm{H}_{2}$ target, but to a lesser extent it is there even for the Ar target where PIE can be expected to be dominant (its contribution is enhanced by a factor of $Z_{T}$ as compared to EIE). In contrast, the full calculations including PIE as well as EIE processes are in quite good agreement with the experimental data (within $1 \sigma-2 \sigma$ ). Moreover, from the comparison it is evident that including the GBI in the EIE calculations is essential to reproduce the experimental data.

It is also of interest to compare the current experimental results for He-like uranium with the ones obtained by us previously for H-like uranium [21]. Similarly to the earlier study (see Fig. 3 in Ref. [21]), we see the decrease of the $K \alpha$ intensity ratios for the $\mathrm{H}_{2}$ target as compared to $\mathrm{Ar}$ which is mainly due to significantly larger EIE cross sections for the $\left[1 s_{1 / 2}, 2 s_{1 / 2}\right]{ }^{3} S_{1}$ and $\left[1 s_{1 / 2}, 2 p_{1 / 2}\right]{ }^{3} P_{1}$ states as compared to the PIE. This is shown in Fig. 5 where the theoretical PIE and EIE cross sections to different $L$-shell levels in He-like uranium are displayed for the collision energy of $218 \mathrm{MeV} / u$ (corresponding to $119.6 \mathrm{keV}$ kinetic energy for an equivelocity electron). As shown in Ref. [33], this dominance of EIE over PIE is a relativistic effect which is pronounced for heavy ions, whereas for light systems the EIE and PIE cross sections are very similar. Another qualitative similarity is the slight increase of the ratios with the collision energy. However, the effect is less pronounced for the present case of He-like uranium as compared to H-like uranium. Here, 


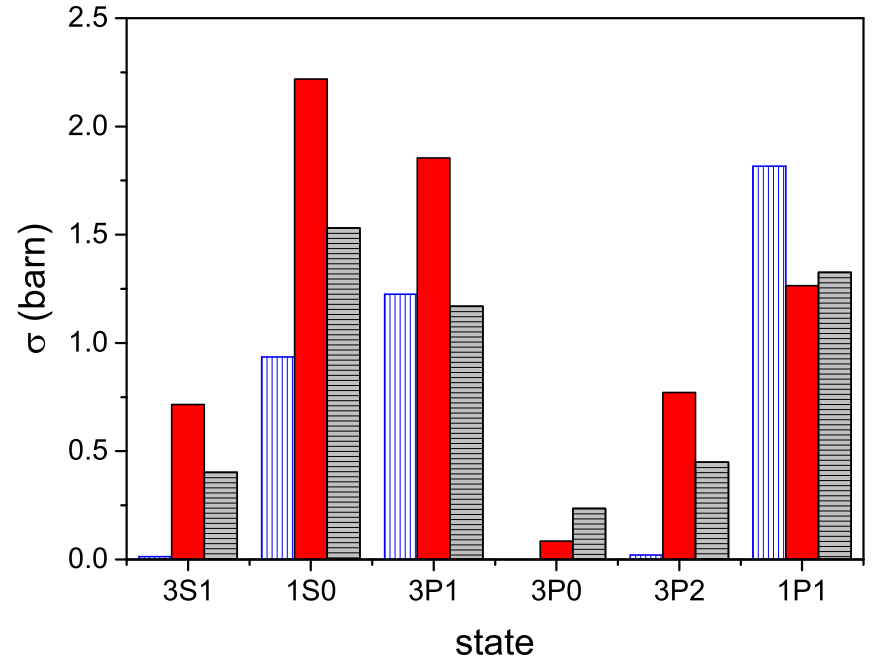

FIG. 5. Theoretical $K$-shell excitation cross sections to different $L$-shell states for $\mathrm{U}^{90+}$ at $218 \mathrm{MeV} / u$ collision energy (corresponding to $119.6 \mathrm{keV}$ electron energy). Blue columns with vertical stripes show PIE results. Solid red columns depict EIE values. In addition, the EIE results without inclusion of the GBI are presented by gray columns with horizontal stripes.

it has to be mentioned that the higher energy in the present measurement was $300 \mathrm{MeV} / u$ as compared to $393.4 \mathrm{MeV} / u$ in the measurement for $\mathrm{H}$-like uranium [21], and thus in principle there the bigger effect can be expected. Taking into account that lower collision energies in both measurements were very close $(218 \mathrm{MeV} / u$ for the He-like case and $212.9 \mathrm{MeV} / u$ for the $\mathrm{H}$-like case), we can also compare the $K \alpha_{1} / K \alpha_{2}$ ratios for the excitation of He-like uranium with the $\operatorname{Ly} \alpha_{1} / \mathrm{Ly} \alpha_{2}$ ratios for the case of $\mathrm{H}$-like uranium, at these energies. Comparing the current results (from Fig. 4) with those from Fig. 3 in Ref. [21], we can see that the $\operatorname{Ly} \alpha_{1} / \mathrm{Ly} \alpha_{2}$ ratios for the $\mathrm{H}_{2}$ target at $212.9 \mathrm{MeV} / u$ are significantly smaller than the $K \alpha_{1} / K \alpha_{2}$ ratios for the same target and basically the same collision energy. Namely, the difference is almost a factor of 2: 0.56(2) for H-like compared to 0.92(2) for He-like. On the one hand, it might be unexpected that the ratio of the cross sections for excitation to different fine-structure levels is so different for He-like and H-like uranium, taking into account especially the similarity of the level structure due to high $Z$ (see Fig. 3). However, this large difference can be explained by the different decay characteristics of He-like uranium as compared to the H-like system. Namely, whereas all the $L$-shell levels $\left(2 s_{1 / 2}, 2 p_{1 / 2}, 2 p_{3 / 2}\right)$ populated by the excitation processes contribute to the intensities of the corresponding Ly $\alpha$ lines in H-like uranium, this is not the case for He-like uranium. Here, the $\left[1 s_{1 / 2}, 2 s_{1 / 2}\right]{ }^{1} S_{0}$ level is strongly populated, in particular, by the EIE process (see Fig. 5), but as mentioned above, it decays via two-photon transition (see Fig. 3) and thus does not contribute to the $K \alpha$ lines [Eq. (2)]. Taking into account the population of this level, in addition to all the other $L$-shell states, gives the theoretical ratio of 0.55 for the population of different fine-structure levels in $\mathrm{He}$ like uranium $\left[\left[1 s_{1 / 2}, 2 p_{3 / 2}\right] /\left(\left[1 s_{1 / 2}, 2 s_{1 / 2}\right]+\left[1 s_{1 / 2}, 2 p_{1 / 2}\right]\right)\right]$, which is in very good agreement with the ratio of $0.56(2)$ for the H-like case.

\section{SUMMARY AND OUTLOOK}

In summary, we studied experimentally and theoretically the $K$-shell excitation of He-like uranium $\left(\mathrm{U}^{90+}\right)$ in collisions with $\mathrm{Ar}$ and $\mathrm{H}_{2}$ targets at 218 and $300 \mathrm{MeV} / u$ energies. By looking at the intensity ratios of $\left(K \alpha_{1} / K \alpha_{2}\right)$ of the subsequent decay photons, we were able to gain access to the proton-impact excitation and to the electron-impact excitation process for the heaviest He-like ion. Relativistic calculations which include both processes, PIE and EIE, provide a good agreement with the experimental data. Moreover, our experimental results clearly demonstrate the importance of including the effect of the GBI in the EIE calculations.

Here, we would like to note that this is not a direct measurement of the EIE process. Nevertheless, taking into account that the $K$-shell excitation of He-like uranium by a $\mathrm{H}_{2}$ target in our collision energy regime can be regarded as an incoherent sum of PIE and EIE processes [2] together with the fact that the theory of PIE has been experimentally tested in a few previous studies [16-18], our current experimental data for the $\mathrm{H}_{2}$ target combined with the PIE calculations provides a quantitative test of the EIE theory for He-like uranium.

In the future, it would be very interesting to experimentally test the predicted difference between the EIE cross sections including the GBI and only the BI, i.e., taking the zero-energy limit for the energy of the exchanged virtual photon between the two electrons [38-40]. Here, it should be emphasized that the difference between using the BI or its generalized version (GBI) in EIE cross sections is usually very small and thus very difficult to be tested experimentally. Only for the excitation of very heavy ions it can be large enough for experimental verification. This, however, would require measuring not only the ratios of the $K \alpha$ intensities, but also their absolute intensities and thus the absolute excitation cross sections with the corresponding precision, which represents a significant challenge.

In addition, these kinds of studies can be extended to investigate the ionization of heavy few-electron ions (via proton or electron impact). Here, recent calculations predict interesting effects when looking at the momenta of the ionized electron ejected in a collision of the ion with a proton and/or an electron [41]. The corresponding experimental setup for electron spectroscopy is already available at the gas target of the ESR [42].

\section{ACKNOWLEDGMENTS}

This work was supported by the Helmholtz Alliance Program of the Helmholtz Association, contract HA216/EMMI "Extremes of Density and Temperature: Cosmic Matter in the Laboratory." The work of C.J.F. was carried out under the auspices of the National Nuclear Security Administration of the U.S. Department of Energy at Los Alamos National Laboratory and supported by Contract No. DE-AC52-06NA25396. S.T. was supported by Deutsche Forschungsgemeinschaft (DFG), Contract No. TA 740 1-1. This work was supported by SPbSU-DFG (Grants No. 11.65.41.2017 and No. STO 346/5-1). R.V.P. acknowledges also the financial support from the Foundation for the advancement of theoretical physics and mathematics "BASIS." 
[1] B. Feinberg, Harvey Gould, W. E. Meyerhof, A. Belkacem, H.-P. Hülskötter, J. R. Alonso, L. Blumenfeld, E. Dillard, N. Guardala, G. F. Krebs, M. A. McMahan, M. J. RhoadesBrown, B. S. Rude, J. Schweppe, D. W. Spooner, K. Street, P. Thieberger, and H. Wegner, Phys. Rev. A 47, 2370 (1993).

[2] B. Najjari and A. B. Voitkiv, Phys. Rev. A 85, 052712 (2012).

[3] R. Dörner, V. Mergel, R. Ali, U. Buck, C. L. Cocke, K. Froschauer, O. Jagutzki, S. Lencinas, W. E. Meyerhof, S. Nüttgens, R. E. Olson, H. Schmidt-Böcking, L. Spielberger, K. Tökesi, J. Ullrich, M. Unverzagt, and W. Wu, Phys. Rev. Lett. 72, 3166 (1994).

[4] W. Wu, K. L. Wong, R. Ali, C. Y. Chen, C. L. Cocke, V. Frohne, J. P. Giese, M. Raphaelian, B. Walch, R. Dörner, V. Mergel, H. Schmidt-Böcking, and W. E. Meyerhof, Phys. Rev. Lett. 72, 3170 (1994).

[5] W. Wu, K. L. Wong, R. Ali, C. Y. Chen, C. L. Cocke, V. Frohne, J. P. Giese, M. Raphaelian, B. Walch, R. Dörner, V. Mergel, H. Schmidt-Böcking, and W. E. Meyerhof, Phys. Rev. A 55, 2771 (1997).

[6] H. Kollmus, R. Moshammer, R. E. Olson, S. Hagmann, M. Schulz, and J. Ullrich, Phys. Rev. Lett. 88, 103202 (2002).

[7] D. Detleffsen, M. Anton, A. Werner, and K. H. Schartner, J. Phys. B 27, 4195 (1994).

[8] K. Reymann, K. H. Schartner, B. Sommer, and E. Träbert, Phys. Rev. A 38, 2290 (1988).

[9] M. Anton, D. Detleffsen, K. H. Schartner, and A. Werner, J. Phys. B 26, 2005 (1993).

[10] N. Stolterfoht, J. Electron Spectrosc. Relat. Phenom. 67, 309 (1993).

[11] N. Stolterfoht, A. Mattis, D. Schneider, G. Schiwietz, B. Skogvall, B. Sulik, and S. Ricz, Phys. Rev. A 51, 350 (1995).

[12] K. Wohrer, A. Chetioui, J. P. Rozet, A. Jolly, F. Fernandez, C. Stephan, B. Brendle, and R. Gayet, J. Phys. B 19, 1997 (1986).

[13] L. Adoui, D. Vernhet, K. Wohrer, J. Plante, A. Chetioui, J. P. Rozet, I. Despiney, C. Stephan, A. Touati, J. M. Ramillion, A. Cassimi, J. P. Grandin, and M. Cornille, Nucl. Instrum. Methods B 98, 312 (1995).

[14] D. Vernhet, J. P. Rozet, K. Wohrer, L. Adoui, C. Stephan, A. Cassimi, and J. M. Ramillion, Nucl. Instrum. Methods B 107, 71 (1996).

[15] B. Brendle, R. Gayet, J. P. Rozet, and K. Wohrer, Phys. Rev. Lett. 54, 2007 (1985).

[16] T. Stöhlker, D. C. Ionescu, P. Rymuza, F. Bosch, H. Geissel, C. Kozhuharov, T. Ludziejewski, P. H. Mokler, C. Scheidenberger, Z. Stachura, A. Warczak, and R. W. Dunford, Phys. Rev. A 57, 845 (1998).

[17] A. Gumberidze, S. Fritzsche, F. Bosch, D. C. Ionescu, A. Krämer, C. Kozhuharov, Z. Stachura, A. Surzhykov, A. Warczak, and Th. Stöhlker, Phys. Rev. A 82, 052712 (2010).

[18] A. Gumberidze, S. Fritzsche, S. Hagmann, C. Kozhuharov, X. Ma, M. Steck, A. Surzhykov, A. Warczak, and T. Stöhlker, Phys. Rev. A 84, 042710 (2011).

[19] M. Kühnel, N. Petridis, D. F. A. Winters, U. Popp, R. Dörner, Th. Stöhlker, and R. E. Grisenti, Nucl. Instrum. Methods A 602, 311 (2009).

[20] N. Petridis, A. Kalinin, U. Popp, V. Gostishchev, Yu. A. Litvinov, C. Dimopoulou, F. Nolden, M. Steck, C. Kozhuharov, D. B. Thorn, A. Gumberidze, S. Trotsenko, S. Hagmann, U. Spillmann, D. F. A. Winters, R. Dörner, T. Stöhlker, and R. E. Grisenti, Nucl. Instrum. Methods A 656, 1 (2011).
[21] A. Gumberidze, D. B. Thorn, C. J. Fontes, B. Najjari, H. L. Zhang, A. Surzhykov, A. Voitkiv, S. Fritzsche, D. Banas, H. Beyer, W. Chen, R. D. DuBois, S. Geyer, R. E. Grisenti, S. Hagmann, M. Hegewald, S. Hess, C. Kozhuharov, R. Martin, I. Orban, N. Petridis, R. Reuschl, A. Simon, U. Spillmann, M. Trassinelli, S. Trotsenko, G. Weber, D. F. A. Winters, N. Winters, D. Yu, and T. Stöhlker, Phys. Rev. Lett. 110, 213201 (2013).

[22] S. Chantrenne, P. Beiersdorfer, R. Cauble, and M. B. Schneider, Phys. Rev. Lett. 69, 265 (1992).

[23] K. L. Wong, P. Beiersdorfer, K. J. Reed, and D. A. Vogel, Phys. Rev. A 51, 1214 (1995).

[24] D. L. Robbins, P. Beiersdorfer, A. Y. Faenov, T. A. Pikuz, D. B. Thorn, H. Chen, K. J. Reed, A. J. Smith, K. R. Boyce, G. V. Brown, R. L. Kelley, C. A. Kilbourne, and F. S. Porter, Phys. Rev. A 74, 022713 (2006).

[25] J. Eichler and Th. Stöhlker, Phys. Rep. 439, 1 (2007).

[26] T. Ludziejewski, Th. Stöhlker, D. C. Ionescu, P. Rymuza, H. Beyer, F. Bosch, C. Kozhuharov, A. Krämer, D. Liesen, P. H. Mokler, Z. Stachura, P. Świat, A. Warczak, and R. W. Dunford, Phys. Rev. A 61, 052706 (2000).

[27] J. Eichler, Nucl. Phys. A 572, 147 (1994).

[28] A. Surzhykov, U. D. Jentschura, Th. Stöhlker, and S. Fritzsche, Phys. Rev. A 74, 052710 (2006).

[29] A. Gumberidze, D. B. Thorn, C. J. Fontes, B. Najjari, H. L. Zhang, A. Surzhykov, A. Voitkiv, S. Fritzsche, D. Banaś, H. Beyer, W. Chen, R. D. DuBois, S. Geyer, R. E. Grisenti, S. Hagmann, M. Hegewald, S. Hess, C. Kozhuharov, R. Märtin, N. Petridis, R. Reuschl, A. Simon, U. Spillmann, M. Trassinelli, S. Trotsenko, G. Weber, D. F. A. Winters, N. Winters, D. Yu, and T. Stöhlker, J. Phys. B: At. Mol. Opt. Phys. 48, 144006 (2015)

[30] W. R. Johnson, D. R. Plante, and J. Sapirstein, Adv. At. Mol. Opt. Phys. 35, 255 (1995).

[31] A. Surzhykov, U. D. Jentschura, Th. Stöhlker, A. Gumberidze, and S. Fritzsche, Phys. Rev. A 77, 042722 (2008).

[32] C. J. Fontes, D. H. Sampson, and H. L. Zhang, Phys. Rev. A 49, 3704 (1994).

[33] B. Najjari and A. B. Voitkiv, Phys. Rev. A 87, 034701 (2013).

[34] D. Bernhardt, C. Brandau, Z. Harman, C. Kozhuharov, A. Müller, W. Scheid, S. Schippers, E. W. Schmidt, D. Yu, A. N. Artemyev, I. I. Tupitsyn, S. Böhm, F. Bosch, F. J. Currell, B. Franzke, A. Gumberidze, J. Jacobi, P. H. Mokler, F. Nolden, U. Spillman, Z. Stachura, M. Steck, and T. Stöhlker, Phys. Rev. A 83, 020701 (2011).

[35] N. Nakamura, A. P. Kavanagh, H. Watanabe, H. A. Sakaue, Y. Li, D. Kato, F. J. Currell, and S. Ohtani, Phys. Rev. Lett. 100, 073203 (2008).

[36] Z. Hu, X. Han, Y. Li, D. Kato, X. Tong, and N. Nakamura, Phys. Rev. Lett. 108, 073002 (2012).

[37] C. J. Bostock and D. V. Fursa, and I. Bray, Phys. Rev. A 80, 052708 (2009).

[38] D. W. Walker, J. Phys. B 8, 760 (1975).

[39] C. J. Fontes, D. H. Sampson, and H. L. Zhang, Phys. Rev. A 47, 1009 (1993).

[40] C. J. Fontes, H. L. Zhang, D. B. Thorn, and A. Gumberidze, in The 17th International Conference on Atomic Processes in Plasmas (APiP), edited by K. Aggarwal and F. Shearer, AIP Conf. Proc. Vol. 1438 (AIP, Melville, NY, 2012), p. 216. 
[41] K. N. Lyashchenko, O. Yu. Andreev, and A. B. Voitkiv, J. Phys. B: At. Mol. Opt. Phys. 51, 055204 (2018).

[42] P.-M. Hillenbrand, S. Hagmann, A. B. Voitkiv, B. Najjari, D. Banaś, K.-H. Blumenhagen, C. Brandau, W. Chen, E.
De Filippo, A. Gumberidze, D. L. Guo, C. Kozhuharov, M. Lestinsky, Yu. A. Litvinov, A. Müller, H. Rothard, S. Schippers, M. S. Schöffler, U. Spillmann, S. Trotsenko, X. L. Zhu, and T. Stöhlker, Phys. Rev. A 90, 042713 (2014). 